\title{
The Effect of Medium Acidity on the Size of Polystyrene Particles in Suspension
}

\author{
${ }^{1}$ Dinar D. Fazullin, ${ }^{2}$ Gennady V. Mavrin, ${ }^{3}$ IInar A. Nasyrov \\ ${ }^{1-3}$ Kazan Federal University \\ Email:denr3@yandex.ru
}

Received: 20 ${ }^{\text {th }}$ August 2019, Accepted: $30^{\text {th }}$ September 2019, Published: $31^{\text {st }}$ October 2019

\begin{abstract}
With the development of industry, there are higher requirements for the quality of process water, as well as the composition of industrial wastewater. To meet the quality requirements, water is increasingly used in membrane methods or a combination of methods with membrane purification methods. Therefore, at present, the actual issue is the development of more effective permeability and retention capacity and inexpensive membranes. Dynamic membranes have a simple method of production. For the formation of such membranes, micro-, nano-particles of a stable suspension of the membrane-forming component are required. In this work, we investigated the dependence of particle size and the stability of a suspension of polystyrene on the acidity of the medium. It is established that, with an increase in the $\mathrm{pH}$ of the suspension to $\mathrm{pH}=8$, an increase in the $\zeta$-potential of the dispersed phase of polystyrene suspension is observed. The maximum values of the $\zeta$-potential of the dispersed phase of a suspension of polystyrene are observed at $\mathrm{pH}=7$ and $\mathrm{pH}$ $=8$. The smallest particle size of the dispersed phase of the suspension is $60.8 \mathrm{~nm}$; it is formed when the $\mathrm{pH}$ value is 5 units. That will reduce the pore size of the membrane in the process of membrane separation of solutions due to the swelling of polymer particles.
\end{abstract}

Keywords

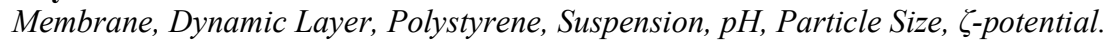

\section{Introduction}

Currently, various types of membrane processes, baromembrane, electro-membrane and diffusion membrane processes with high cleaning efficiency and high productivity are used in water purification and water treatment processes. Of the membrane processes, the simplest and most effective are baromembrane processes, such as ultrafiltration, nanofiltration and reverse osmosis, each of which is used depending on the tasks.

Membranes should have the following properties: high permeability, selectivity, unchanged characteristics during operation and storage, low cost, should be resistant to the action of shared solutions and should have sufficient mechanical strength.

Dynamic membranes satisfy all these requirements - these are composite membranes, which are formed by the formation on the surface of a porous base of a semipermeable layer from suspended microparticles or dissolved substances present in the solution. For the formation of dynamic membranes, colloidal particles, neutral organic polymers, organic and inorganic polyelectrolytes are suitable [1]. The service life of dynamic membranes is almost unlimited. With mechanical damage, they can self-repair, since a new semi-permeable layer is deposited on the substrate.

In [2-3], the authors used polyaniline as the surface layer of membranes. In the first work, polyaniline was applied by electrospinning followed by polymerization on the surface of nanofibers. The resulting membranes were used to remove chromium, the membrane efficiency was more than $70 \%$. Dynamic adsorption tests were also carried out at various flow rates and volumes of chromium in water. In the second work, a polyaniline layer was obtained by polymerization of anilian hydrochloride over the surface of a substrate. The membrane was used to remove iron ions, the cleaning efficiency of iron ions was $80-90 \%$.

In another work [4], a suspension of $\mathrm{T}_{\mathrm{i}} \mathrm{O}_{2}$ particles in a solution of polyvinyl alcohol was used to obtain a dynamic layer. The membrane was used to separate oil-in-water emulsions; the oil removal efficiency from the emulsion was $99.8 \%$ at a working pressure of $0.1 \mathrm{MPa}$. The membrane also showed excellent resistance to fouling and the possibility of processing.

Strong and inert material for obtaining a dynamic layer is polystyrene. In [5], the effect of the chemical composition of water (hydrogen index, dissolved organic carbon, and divalent cations) on the properties of a polystyrene suspension was investigated. The effect of the chemical properties of water on the functionality of the surface of polystyrene particles, on stability, on particle aggregation is investigated. Suspension toxicity was also investigated. For the study, the authors obtained model polystyrene latex nanoparticles (PLNP) with three different functional groups, namely unmodified (uPLNP), amine-modified (aPLNP) and carboxyl-modified (cPLNP). The results showed that the presence of dissolved organic carbon increases the surface charge and has little effect on the particle size distribution of polystyrene in aqueous suspensions. The authors determined that the presence of divalent cations of calcium and magnesium reduces the surface charge and increases the particle size of polystyrene. The combined presence of dissolved organic carbon and divalent cations in an aqueous suspension increases the degree of aggregation of polystyrene particles. It was also found that the hydrogen index significantly affects the stability of polystyrene particles in suspension. Observations using electron 
scanning microscopy showed that increased aggregation of polystyrene particles in the presence of dissolved organic carbon and a divalent cation is caused by the formation of a bridge. The toxicity of the suspension showed that the polystyrene suspension showed acute toxicity to Daphnia magna Straus. And polystyrene suspensions modified with amine and carboxyls had a higher toxic effect on Daphnia magna Straus.

The issue of uniform distribution of polystyrene particles on the surface was studied in [6]. Acoustic tweezers facilitate the manipulation of microparticles using sound waves. In [6], a modern system is presented in which an acoustic lens was used to develop a microacoustic trap for assembling microparticles in 3D. Model particles, polystyrene balls with a diameter of $2 \mu \mathrm{m}$ in suspension, were driven by acoustic pressure to form a monolayer at distances determined by the wavelength above the substrate, determined by the focal point of the acoustic lens. The sensor worked at a frequency of $89 \mathrm{MHz}$, pulses with a repetition rate of $2 \mathrm{~Hz}$. The acoustic effect led to a two-dimensional assembly of microparticles into tightly packed layers with a width of more than 80 microns. This method of particle distribution provides maneuverability when driving or assembling objects of micrometer scale using continuous or pulsed focused pressure of acoustic radiation.

In [7], a method for producing monolayers of microparticles in the form of spheres from polystyrene particles was described. Particles were obtained by the authors from microspheres consisting of a polystyrene core and a shell enriched in polyglycidol by stretching polyvinyl alcohol films that contain embedded polystyrene particles. The elongated films were dissolved in water, and the microspheroids were subsequently removed and purified from polyvinyl alcohol. Particles were deposited on silicon wafers from a suspension in ethanol. The particle concentration and suspension volume were the same in each experiment. Evaporation of ethanol resulted in the formation of layers of spherical particles packed in colloidal nematic crystals. The orientation of the bands after the evaporation of ethanol was perpendicular to the threephase interface (silicon-ethanol-air) along the silicon substrate. The distribution of nematic type spheroids in the bands was the dominant structure, which made it possible to more densely pack particles into colloidal crystals.

The aim of this work is to obtain a suspension of polystyrene with a minimum particle size of the dispersed phase to use particles as a dynamic layer of an ultrafiltration membrane.

\section{Methods}

To obtain a dynamic membrane with a surface layer of polystyrene, a suspension of polystyrene in an aqueous solution of acetone was prepared. The suspension was obtained by dissolving polystyrene in acetone ( $5 \mathrm{~g}$ in $100 \mathrm{ml})$, after which distilled water was added to the solution in a 1:1 ratio and stirring was performed without air. As a result of adding water, polystyrene particles are formed [8-10].

In order to obtain a suspension of polystyrene with the smallest particle size, a solution of hydrochloric acid was added to the suspension to acidify, and a solution of sodium hydroxide was added to increase alkalinity. As a result, suspensions were obtained with hydrogen values equal to $2,4,5,6,7,8,9 \mathrm{pH}$ units.

The particle size of the dispersed phase of the suspensions was determined by dynamic light scattering, and the $\zeta$-potential was determined by light scattering with phase analysis using a Nano Brook Omni analyzer. To study the characteristics of the initial samples, the following parameters were taken from the available databases: solvent - an aqueous solution of acetone; viscosity $-0.89 \times 10^{-6} \mathrm{~m}^{2} \cdot \mathrm{s}^{-1}$; scattering angle $-90^{\circ}$; the measurement duration is 3 minutes, the number of measurements is 3 . Next, the system automatically optimizes the parameters and sets the temperature, the starting point of the measurement. The device gives the results: information on the intensity of the particle size distribution of the emulsions - diameter (nm), intensity (\%), peak width, $\zeta$-potential of particles, particle mobility.

The acidity of the medium was measured by the potentiometric method using an Anion 4100 ionomer / $\mathrm{pH}$ meter using an electrode system consisting of an indicator electrode and a reference electrode.

\section{Results and Discussion}

In order to obtain a suspension of polystyrene in an aqueous solution of acetone with the smallest particle size, suspensions were prepared with $\mathrm{pH}$ values from 2 to $9 \mathrm{pH}$ units. The $\mathrm{pH}$ value of the initial emulsion was 6 units. Table 1 presents the value of the $\zeta$-potential of the dispersed phase of a suspension of polystyrene in an aqueous solution of acetone, depending on the hydrogen index.

\begin{tabular}{|c|c|c|c|}
\hline No. & Hydrogen indicator of polystyrene suspension, $\mathrm{pH}$ & $\zeta$-potential, $\mathrm{mV}$ & Mobility of particles, $\mathrm{mV}$ \\
\hline 1 & 2 & $-7,0$ & $-0,6$ \\
\hline 2 & 3 & $-10,6$ & $-0,8$ \\
\hline 3 & 5 & $-24,3$ & $-1,9$ \\
\hline 4 & 6 & $-42,7$ & $-3,3$ \\
\hline 5 & 7 & $-58,5$ & $-4,6$ \\
\hline 6 & 8 & $-59,2$ & $-4,6$ \\
\hline 7 & 9 & $-55,7$ & $-4,4$ \\
\hline
\end{tabular}

Table 1: The Values of the $\zeta$-potential of the Dispersed Phase of a Suspension of Polystyrene in an Aqueous Solution of Acetone. 
According to table 1, with an increase in the $\mathrm{pH}$ of the suspension to $\mathrm{pH}=8$, an increase in the $\zeta$-potential of the dispersed phase of the polystyrene suspension is observed. The $\zeta$-potential of the dispersed phase is an indicator of the stability of the suspension: the larger the absolute value of the $\zeta$-potential, the stronger the particles of the dispersed phase of the suspension repel each other, preventing the aggregation and coalescence of particles. The maximum $\zeta$-potential of the dispersed phase of the polystyrene suspension is observed at $\mathrm{pH}=7$ and $\mathrm{pH}=8$.

The following are the results of a study of the particle size of the suspension. Figures 1-5 show graphs of the particle size distribution of the dispersed phase of a polystyrene suspension.

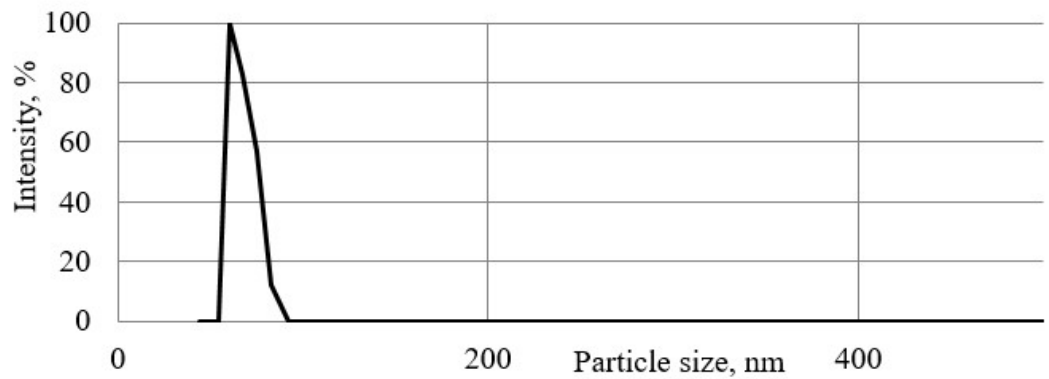

Figure 1: Graph of Particle Size Distribution of Polystyrene Suspension in an Aqueous Solution of Acetone at pH $=5$.

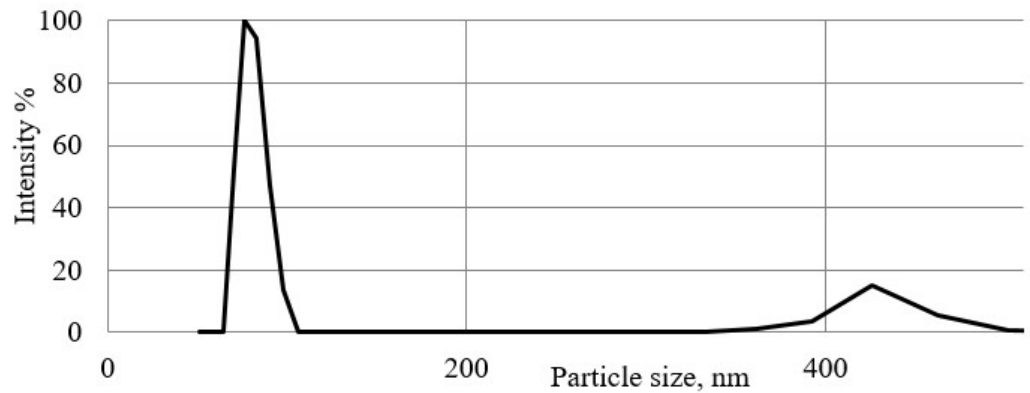

Figure 2: Graph of Particle Size Distribution of the Suspension of Polystyrene in an Aqueous Solution of Acetone at $\mathbf{p H}=6$.

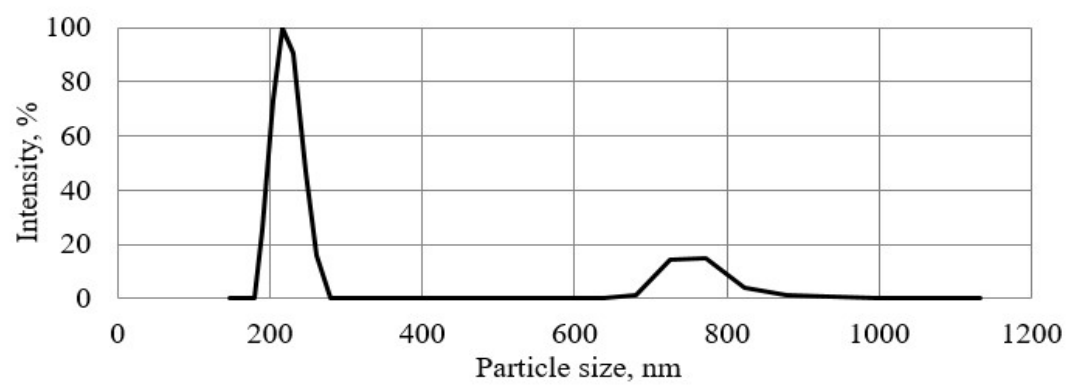

Figure 3: Graph of Particle Size Distribution of the Suspension of Polystyrene in an Aqueous Solution of Acetone at $\mathbf{p H}=7$.

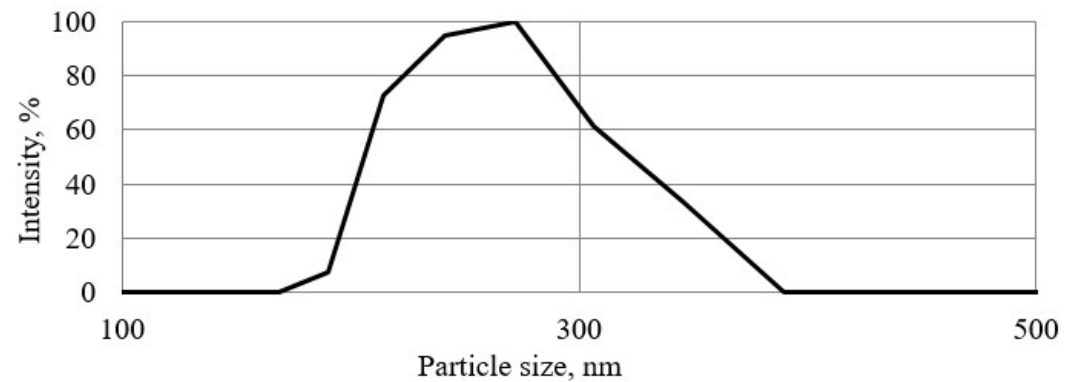

Figure 4. Graph of Particle Size Distribution of Polystyrene Suspension in an Aqueous Solution of Acetone at pH $=8$. 


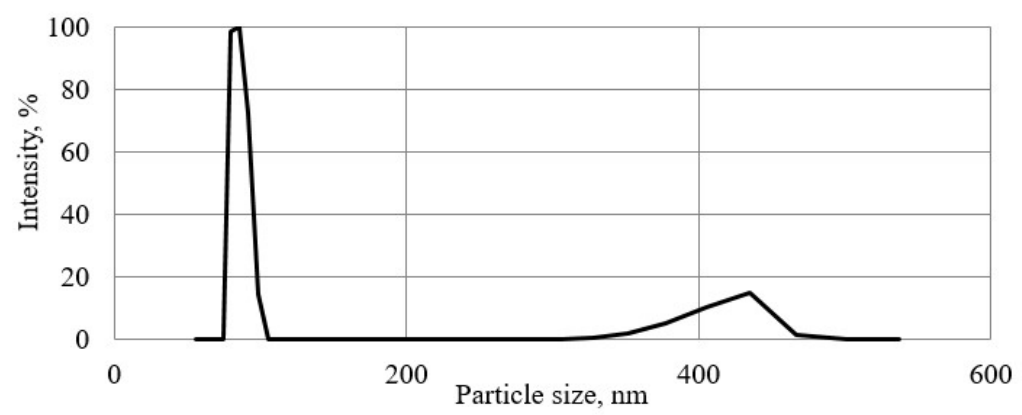

Figure 5. Graph of Particle Size Distribution of the Suspension of Polystyrene in an Aqueous Solution of Acetone at $\mathbf{p H}=9$.

Table 2 presents the particle sizes of the dispersed phase of the suspension.

\begin{tabular}{|c|c|c|}
\hline No. & Hydrogen indicator of polystyrene suspension, $\mathrm{pH}$ & Size of polystyrene particles in suspension, $\mathrm{nm}$ \\
\hline 1 & 2 & $>10000$ \\
\hline 2 & 3 & $>1000$ \\
\hline 3 & 5 & $60,8-83,1$ \\
\hline 4 & 6 & $70,4-97,6 ; 361,9-502,2$ \\
\hline 5 & 7 & $190,5-261,9 ; 680,5-935,5$ \\
\hline 6 & 8 & $190,1-345,5$ \\
\hline 7 & 9 & $79,8-98,7 ; 328-466,9$ \\
\hline
\end{tabular}

Table 2: The Particle Size of the Dispersed Phase of the Suspension, Depending on the Acidity of the Medium.

According to table 2, the minimum values of PS particle size are formed when the $\mathrm{pH}$ value of the suspension is $\mathrm{pH}=5$. At values of the hydrogen index below $\mathrm{pH}=5$, the destruction of the suspension and aggregation of PS particles to sizes greater than $10 \mu \mathrm{m}$ are observed. With an increase in the hydrogen index to $\mathrm{pH}=8$, the particle sizes of the dispersed phase of the suspension increase, and at $\mathrm{pH}=9$, the particle size of PS decreases. At these values of the hydrogen index, the minimal absolute values of the $\zeta$-potential of the polystyrene suspension are also observed. The smallest particle size of the dispersed phase of the suspension of $60.8 \mathrm{~nm}$, corresponds to a $\mathrm{pH}$ value of 5 units.

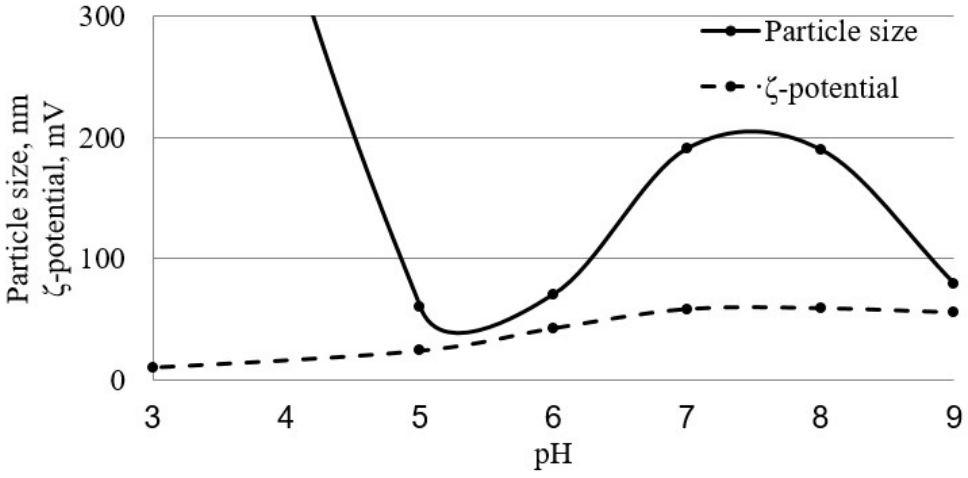

Figure 6: Minimum Particle Sizes and $\zeta$-potential Values of a Polystyrene Suspension Depending on the pH of the Medium.

According to Figure 6, the minimum particle sizes of polystyrene are formed in suspension in the range of a hydrogen index from 5 to $6 \mathrm{pH}$. And the absolute values of the $\zeta$-potential of the polystyrene suspension decrease with increasing acidity of the medium.

\section{Summary}

In order to obtain a suspension of polystyrene in an aqueous solution of acetone with the smallest particle size, suspensions were prepared with hydrogen values from 2 to $9 \mathrm{pH}$ units.

According to table 1, with an increase in the $\mathrm{pH}$ of the suspension to $\mathrm{pH}=8$, an increase in the $\zeta$-potential of the dispersed phase of the polystyrene suspension is observed. The maximum $\zeta$-potential of the dispersed phase of the polystyrene suspension is observed at $\mathrm{pH}=7$ and $\mathrm{pH}=8$. 
The smallest particle size of the dispersed phase of a suspension of $60.8 \mathrm{~nm}$ is formed when the pH value is 5 units with an increase in the hydrogen index to $\mathrm{pH}=8$, the particle sizes of the dispersed phase of the suspension increase, and at $\mathrm{pH}=9$, the particle size of PS decreases.

\section{Conclusions}

According to the results of the study, it was found that the hydrogen index affects the particle size of polystyrene in suspension. To form a dynamic membrane layer according to the results of the study, it was found that it is necessary to use a suspension of polystyrene with an acidity of $\mathrm{pH}=5$, which will reduce the pore size of the membrane during membrane separation of solutions at $\mathrm{pH}$ values of more than $5 \mathrm{pH}$ units due to swelling of the polymer particles. Thus, according to Fig. 1, upon separation of the solution with $\mathrm{pH}=6$, PS particles should increase by $15 \%$, and at $\mathrm{pH}$ values of $7-8$, the $\mathrm{pH}$ should be more than 3 times.

\section{Acknowledgements}

The work is performed according to the Russian Government Program of Competitive Growth of Kazan Federal University. The work was supported by a grant from the President of the Russian Federation for state support of young Russian scientists - PhDs (MK-1107.2019.8).

\section{References}

[1] Lavrenchenko A.A., Lazarev S.I. Investigation of the kinetic characteristics of dynamic membranes in the process of ultrafiltration purification of industrial solutions of biochemical production. 2015, Vol. 3. P. 28-33.

[2] Guilherme Dognani, Pejman Hadi, Hongyang Ma. Effective chromium removal from water by polyaniline-coated electrospun adsorbent membrane. Chemical engineering journal. 2019. Vol. 372. P. 341-351.

[3] Fazullin D.D., Mavrin G.V., Shaikhiev I.G, Dvoryak S.V. The properties of cation-exchange membranes PTFEPANI. Research Journal of Pharmaceutical, Biological and Chemical Sciences. 2015. Vol. 6. Issue 6. P. $1793-1798$.

[4] Ze Li, Zhen-Liang Xu, Ben-Qing Huang. Three-channel stainless steel hollow fiber membrane with inner layer modified by nano-TiO2 coating method for the separation of oil-in-water emulsions. Separation and purification technology. 2019. Vol. 222 P. 75-84.

[5] Zhang Fan, Wang Zhuang, Wang Se. Aquatic behavior and toxicity of polystyrene nanoplastic particles with different functional groups: Complex roles of $\mathrm{pH}$, dissolved organic carbon and divalent cations. Chemosphere. 2019. Vol. 228. P.195-203.

[6] Vyas Varun, Lemieux Michael, Knecht David A., Kolosov Oleg V., Huey Bryan D. Micro-Acoustic-Trap (mu AT) for microparticle assembly in 3D. Ultrasonics sonochemistry. 2019. Vol. 57. P. 193-202. DOI: 10.1016/j.ultsonch.2019.05.010.

[7] Mickiewicz Damian, Basinska Teresa, Gosecka Monika, Gadzinowski Mariusz et al. Colloidal liquid crystal type assemblies of spheroidal polystyrene core/polyglycidol-rich shell particles (P[S/PGL]) formed at the liquid-siliconair interface by a directed dewetting process. Polymers for advanced technologies. 2019. Vol. 30. Issue 7. P. 17241731. DOI: $10.1002 /$ pat.4604

[8] Fazullin D.D., Mavrin G.V., Shaikhiev I.G. Ultrafiltration of Oil-in-Water Emulsions with a Dynamic NylonPolystyrene Membrane. Petroleum Chemistry. 2018. Vol. 58. No. 2. P. 145-151.

[9] Fazullin D.D., Mavrin G.V., Shaikhiev I.G. Exposure to microwave radiation on thin-film polymer membranes. Electronic processing of materials. 2019. Vol. 55. No. 3. P. 58-65 DOI: 10.5281/zenodo.3244416

[10] Fazullin D.D., Mavrin G.V., Shaikhiev I.G., I. R. Nizameev Microwave Stabilization of a Dynamic Membrane. Membranes and Membrane Technologies. 2019. Vol. 1, Issue 1, P. 1-6. 